\title{
The effect of boarfish protein hydrolysate on postprandial glycaemic response and satiety in healthy adults
}

\author{
W. Crowe ${ }^{1}$, C.M. McLaughlin ${ }^{2}$, P.J. Allsopp ${ }^{1}$, M.M. Slevin ${ }^{1}$, P.A. Harnedy ${ }^{3}$, Y. Cassidy ${ }^{1}$, \\ J. Baird ${ }^{1}$, M. Devaney ${ }^{1}$, R.J. Fitzgerald ${ }^{3}$, F.P.M. O’Harte ${ }^{2}$ and E.M. McSorley ${ }^{1}$ \\ ${ }^{1}$ Nutrition Innovation Centre for Food and Health, School of Biomedical Sciences, Ulster University, Coleraine, \\ Co. Derry, BT52 1SA, ${ }^{2}$ The SAAD Centre for Pharmacy \& Diabetes, School of Biomedical Sciences, Ulster \\ University, Coleraine, Co. Derry, BT52 1SA and ${ }^{3}$ Department of Biological Sciences, University of Limerick, \\ Limerick, Ireland
}

There have been increased efforts to find an added-value application for the currently underutilised pelagic fish species, Boarfish $(\text { Capros aper })^{(1)}$. Recent in vitro and in vivo research has demonstrated that fish protein hydrolysate (BPH) has a beneficial effect on glycaemic control and reduced food intake ${ }^{(2)}$. This study aims to investigate the effect of consuming boarfish on postprandial glycaemic control in human participants.

A randomised controlled human intervention crossover study was undertaken in a cohort of healthy adults $(\mathrm{n}=20)$ to investigate the effect of consuming a BPH (3.5 g) drink on postprandial glycaemic control in comparison to a control drink. Blood samples were collected before (0 mins) and after the test drink was consumed over a 3-hour period (15, 30, 60, 90,120 and 180 mins). Collected blood samples were analysed for biomarkers of satiety (ghrelin, leptin) and glycaemic control (glucose, insulin, glucagon-like peptide 1 (GLP-1)). Visual analogue scales (VAS) were completed at each time point by participants to provide a subjective measure of their satiety.

Paired T test analysis revealed no significant difference between the area under the curve (AUC) for insulin or glucose, when the effect of BPH consumption was compared to the standard drink. A significant increase in satiety rating by $11.1 \%$ at 180 minutes $(P=$ 0.034) was reported following BPH consumption in comparison to the control drink (Fig. 1).

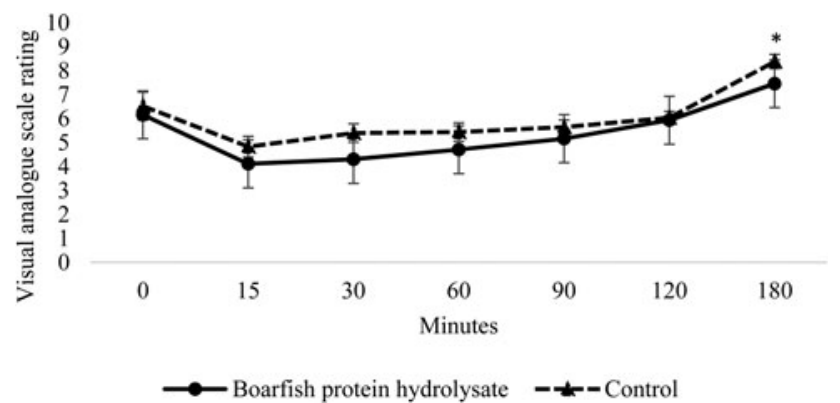

Fig. 1. Subjective hunger ratings over 3 hours by participants consuming boarfish protein hydrolysate and control.

This study showed that consuming $3.5 \mathrm{~g}$ of $\mathrm{BPH}$ had no effect on biomarkers of satiety or glycaemic control, albeit a higher dose may be required to see an effect. The BPH may have satiating effects which warrant further investigation.

1. Hayes M, Mora L, Hussey K, Aluko RE (2016) Innov Food Sci Emerg Technol 37, 253-60.

2. Harnedy PA, Parthsarathy V, McLaughlin CM et al. (2018) J Func Foods 40, 137-45. 